\title{
ASAPe: uma Arquitetura para Sensoriamento Preditivo em Computação Vestível
}

\author{
Michelle G. Cacais ${ }^{1}$, Leonardo O. Moreira ${ }^{1}$, Weslley L. Caldas ${ }^{1}$, \\ Danielo G. Gomes ${ }^{1}$ \\ ${ }^{1}$ Universidade Federal do Ceará (UFC) - Fortaleza, CE - Brasil \\ [michellecacais, weslleylc] @alu.ufc.br, [leoomoreira, danielo]@ufc.br
}

\begin{abstract}
Among the several concepts associated with Internet of Things (IoT), we highlight wearable computing, which involves body sensing to capture data from people who are "wearing"the sensor. This paper presents ASAPe, an architecture for predictive sensing in wearable computing. Techniques and strategies for implementing our proposal are discussed and evaluated in this proposal. The results achieved so far indicate that ASAPe can be very useful both in real-time data collection contexts as well as in massive amounts of data. The main contribution is a new paradigm for working components and prediction in Wearable Computing.
\end{abstract}

Resumo. Dentre os vários conceitos associados à Internet das Coisas (IoT), destacamos a computação vestivel, a qual envolve sensoriamento corporal para captar dados das pessoas que estiverem "vestindo"o sensor. Este artigo apresenta ASAPe, uma arquitetura para sensoriamento preditivo em computação vestível. Técnicas e estratégias de implementação da nossa proposta são discutidas e avaliadas nesta proposta. Os resultados alcançados até o presente momento indicam que a ASAPe pode ser muito útil tanto em contextos de coleta de dados em tempo real como também em quantidade massiva de dados. A principal contribuição é fornecer um novo paradigma para trabalhar componentes e predição em Computação Vestível.

\section{Introdução}

Na última década, temos visto uma significativa proliferação de objetos físicos passíveis de conexão à Internet. Com o paradigma da Internet das Coisas (IoT), os chamados objetos inteligentes oferecem informações sensoriadas do seu entorno físico em uma comunicação objeto-a-objeto [Liu and Zhou 2012]. O aspecto vago do termo "Coisas"ao mesmo tempo que dificulta a própria definição do conceito de IoT, dá uma ideia clara de sua heterogeneidade e de seu potencial de aplicação praticamente ilimitado. Dentre as várias possibilidades e vertentes da IoT, destacamos a computação vestível.

Com o advento da computação ubíqua/pervasiva, a noção de computação vestível dos anos 1980-1990 expandiu-se e hoje encontra-se incorporada ao conceito de IoT. A pesquisa e o desenvolvimento em computação vestível têm propiciado soluções mais funcionais e atrativas aos seus usuários em potencial, grande parte delas voltada para as áreas da saúde [Conjeti et al. 2012], [Molina-Markham et al. 2014] [Gope and Hwang 2016], 
fitness, [Pels et al. 2014] [Rocha et al. 2015], [Salehizadeh et al. 2016], esportes [Nguyen et al. 2015], [Spelmezan 2012], como também para a área social [Kan et al. 2015] [Chen et al. 2015].

A computação vestível também permite ao usuário permanecer conectado e monitorado constantemente através de sua roupa ou de outro acessório sensor acoplado ao corpo. Dependendo do propósito, este recurso pode ser adequado quando o usuário deve ser monitorado para evitar eventos indesejados ou acidentes. Apesar da literatura recente incluir uma variedade de soluções para detecção preventiva em computação vestível ([Chen et al. 2015], [Forkan and Khalil 2016], [Jaimes et al. 2017] [Kang et al. 2015]), não há uma arquitetura ou framework específico para análise de dados corporais e predição em Computação Vestível.

Desse modo, apresentamos ASAPe, uma arquitetura para sensoriamento preditivo para computação vestível. O intuito é criar um mecanismo para organizar componentes em infraestruturas de computação vestível, ajudando com a reutilização e padronização. Além disso, o principal foco é agregar uma camada de predição para dar suporte à análise de dados e prevenção de eventos indesejados com base nos dados corporais do usuário.

\section{Trabalhos Correlatos}

Alguns trabalhos na área de Computação Vestível foram desenvolvidos para resolver problemas relacionados à essa temática. Usamos strings de busca como: "wearable computing frameworks", "body sensor networks framework", "architectures for wearable computing"e "predictive sensing in wearable computing", nas bases de dados IEEE ${ }^{1}, \mathrm{ACM}^{2}$ $\mathrm{e} \mathrm{ISI}^{3}$, entre 2014 e 2017. Aqui, listamos os que lidam com detecção preditiva em Wearable Computing, frameworks que ajudam com a heterogeneidade de componentes e arquiteturas wearable projetados para Computação Vestível.

O sensoriamento preditivo é usado em Computação Vestível principalmente para evitar eventos fisiológicos indesejados. Em [Forkan and Khalil 2016], os autores usaram Hidden Markov Model (HMM) a fim de identificar eventos fisiológicos de um paciente. O trabalho visto em [Jaimes et al. 2017] fala sobre o uso de HMM e previsão de suavização exponencial para evitar episódios de estresse. Em [Kan et al. 2015], os autores forneceram um trabalho sobre detecção preditiva para situações de saúde urgentes em tempo real.

Os frameworks voltados para Computação Vestível tem como objetivo fornecer abstração da heterogeneidade de infraestruturas wearable. $\mathrm{O}$ trabalho visto em [Gheith et al. 2016] é sobre o IBM Bluemix, uma nuvem oferecida pela IBM. É uma plataforma para aplicações móveis baseadas na nuvem. Em [Uddin et al. 2015] os autores forneceram um quadro genérico para monitorar continuamente as atividades diárias. Em [Galzarano et al. 2016] criou-se uma ferramenta específica de domínio denominada SPINE 2, para prototipagem rápida de redes sem fio de sensores corporais (WBSN).

As arquiteturas voltadas para Computação Vestível resolvem problemas relacionados à organização dos componentes envolvidos em infraestruturas werable. O traba-

\footnotetext{
${ }^{1}$ http://ieeexplore.ieee.org/Xplore/home.jsp

${ }^{2}$ https://dl.acm.org/

${ }^{3}$ https://pcs.webofknowledge.com/
} 
lho visto em [Abdali-Mohammadi et al. 2015] é sobre uma arquitetura para a construção de sistemas vestíveis em aplicações médicas para observar situações críticas. Já [Chen et al. 2015] apresenta AIWAC, uma arquitetura para computação vestível, voltada para a interação afetiva. O trabalho [Wang et al. 2016] é relacionado a uma arquitetura para padronizar o desenvolvimento do tecido inteligente adequado para roupas inteligentes e WBSN.

\subsection{Comparação}

A detecção preditiva para sensores corporais tem sido usada em maioria para princípios de saúde. As arquiteturas encontradas na literatura não são usadas para detecção preditiva. Desta forma, escolhemos duas características que distinguem os trabalhos relacionados da nossa proposta: se o trabalho propõe algum modelo para a organização dos componentes em uma aplicação vestível (Organização), e se envolve previsão (Predição). A Tabela 1 dá uma visão geral sobre a literatura relacionada, bem como destaca algumas diferenças de nossa proposta.

Tabela 1. Resumo comparativo de trabalhos correlatos.

\begin{tabular}{|l|c|c|}
\hline Referência & Organização & Predição \\
\hline \hline [Forkan and Khalil 2016] & & $\sqrt{ }$ \\
\hline [Jaimes et al. 2017] & & $\sqrt{ }$ \\
\hline [Kan et al. 2015] & & $\sqrt{ }$ \\
\hline [Gheith et al. 2016] & $\sqrt{ }$ & \\
\hline [Uddin et al. 2015] & $\sqrt{ }$ & \\
\hline [Galzarano et al. 2016] & $\sqrt{ }$ & \\
\hline [Abdali-Mohammadi et al. 2015] & $\sqrt{ }$ & \\
\hline [Chen et al. 2015] & $\sqrt{ }$ & \\
\hline [Wang et al. 2016] & $\sqrt{ }$ & \\
\hline Nossa proposta & $\sqrt{ }$ & $\sqrt{ }$ \\
\hline
\end{tabular}

\section{ASAPe}

O Arcabouço para Sensoriamento Preditivo em Computação Vestível(ASAPe) é uma arquitetura para suportar aplicativos wearable que requerem gerenciamento proativo. Nesse sentido, nossa proposta pode ajudar no desenvolvimento de sistemas que precisam de algum ajuste de uma situação atual para evitar eventos adversos. ASAPe é composto por componentes de software que realizam serviços para um único componente residente no aplicativo cliente. Uma visão geral da arquitetura do sistema proposto pode ser vista na Figura 1.

- Agent: sensores responsáveis pela obtenção de dados corporais e ambientais

- Coordinator Service: camada responsável por capturar dados dos sensores e enviá-lo para o Forecast Service. Ele também chama o Event Rules Database, para acessar as regras de ativação, notificar o usuário e propor mudanças no ambiente.

- ASAPe Application Interface: especifica o conjunto de ações que podem ser executadas quando ocorre um evento. 


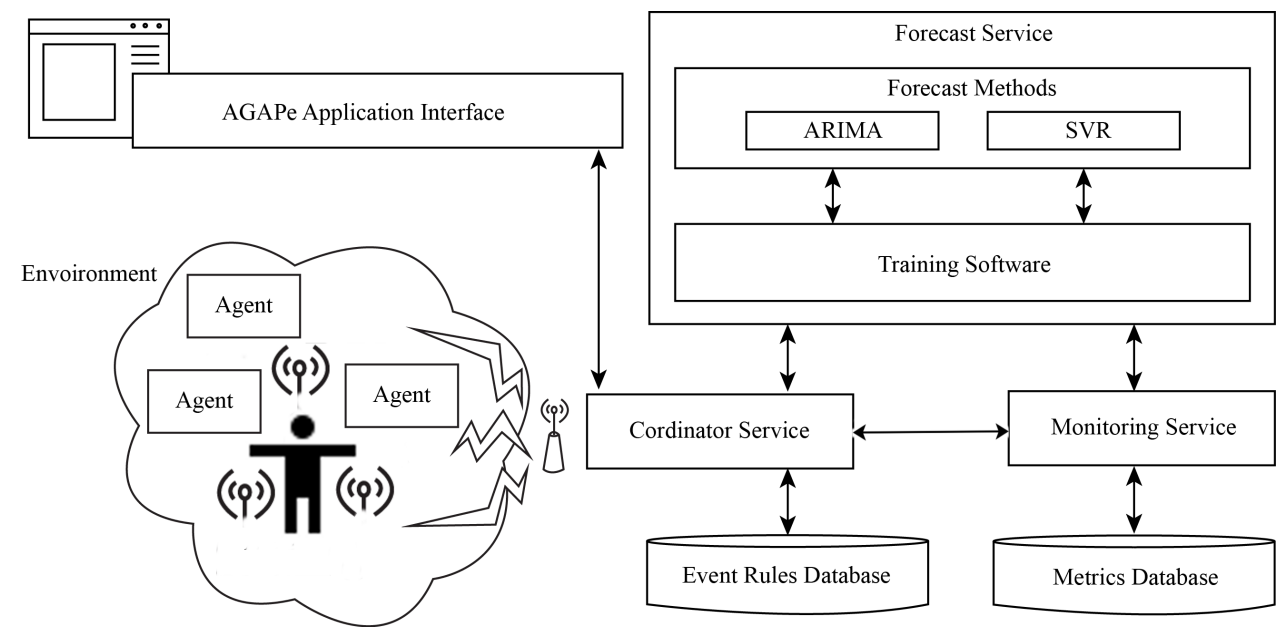

Figura 1. Arquitetura da ASAPe.

- Event Rules Database: é um banco de dados relacional que mantém as regras de ativação dos eventos.

- Metrics Database: é o banco de dados usado para salvar os valores capturados pelo componente Agente. Esses dados podem ser armazenados para definir padrões.

- Monitoring Service: essa camada recebe dados do Coordinator Service e os grava no Metrics Database.

- Forecast Service: é o componente que faz a previsão. Ele recebe dados do Coordinator Service, que, por sua vez, obtém dados do Event Rules Database, e Monitoring Service, que o leva do Metrics Database. Faz uso de três componentes.

- Training Software: é responsável por tomar os dados de monitoramento e aplicar um dos modelos (ARIMA ou SVR) para obter quadros de predição.

- ARIMA: é um algoritmo estatístico que faz o ajuste de dados de séries temporais para prever pontos futuros.

- SVR: um método supervisionado de aprendizagem de máquina para o reconhecimento de padrões, usado principalmente para análise de classificação e regressão.

\subsection{Visão geral dos componentes da ASAPe}

Os componentes na primeira camada wearable são conhecidos como Sensores de Área de Corpo (Wearable Body Area Sensors (WBAS)), composto por hardware de sensores com potência de computação limitada e largura de banda de comunicação. Um gateway (por exemplo, smartphone ou tablet) envia dados para um ponto final (por exemplo, uma infraestrutura de computação em nuvem) que fornece gerenciamento de dados e oferece suporte a funcionalidades avançadas de mineração de dados, aprendizado de máquina e análise de dados médicos [Hiremath et al. 2014].

A área do corpo monitorado pode possuir uma coleção de nós sensores, ou nós de computador, como eletrocardiograma (ECG), eletromiografia (EMG), eletroencefalografia (EEG), pressão arterial (BP), etc. Para obter essas métricas sensoriais e reagir com os objetos no ambiente, o componente Agent pode ser implantado em nós de sensores 
ou nós de computador. O Agent também pode utilizar alguns desses sensores, sistemas operacionais ou aplicativos para interagir ou reagir aos sensores na pessoa monitorada.

Cada bit de dados é enviado para o componente Coordinator Service, que os envia para o Monitoring Service e, finalmente, registra os dados em um banco de dados relacional, mostrado como o componente Metrics Database na arquitetura proposta. Além de receber dados do componente Agent, o Coordinator Service resolve as requisições do lado cliente e tem a responsabilidade de comunicar e coordenar o Monitoring Service, o Forecast Service e Event Rules Database. O Coordinator Service é executado continuamente e realiza eventos que foram especificados pela ASAPe Application Interface.

O aplicativo cliente utiliza a ASAPe Application Interface para especificar um conjunto de ações que podem ser executadas quando ocorre um determinado evento ou sua ocorrência foi prevista. Os eventos são ativados quando um conjunto de métricas atinge, ultrapassa ou chega perto de um determinado valor. As ações que podem ser realizadas quando ocorre um evento são especificadas por instruções codificadas pelo desenvolvedor e salvas no banco de dados Event Rules Database.

Existem dois tipos de bancos de dados em nossa arquitetura: Event Rules Database e Metrics Database. O Banco de Dados Relacional salva os valores capturados pelos sensores na área do corpo e é mostrado como o Banco de Dados de Metrics. Esses dados podem ser usados para prever séries temporais e serem armazenados para definir padrões.

O componente Event Rules Database é um banco de dados relacional que mantém as regras para a ativação de eventos reativos ou preditivos. Uma regra de ativação reativa é adicionada sempre que o sistema de monitoramento baseado na arquitetura proposta lê um ou mais valores de uma ou mais das métricas determinadas pelo aplicativo do cliente. O sistema implantado baseado na arquitetura utiliza métodos de predição para prever o instante no tempo em que uma métrica atingirá um certo valor, executando ajustes no ambiente e impedindo a ocorrência de valores ou fatores indesejados, nas métricas ou no meio ambiente.

O componente Event Rules Database é chamado continuamente pelo Coordinator Service para obter acesso às regras de ativação dos eventos do aplicativo cliente. Para esse fim, o Coordinator Service consulta o sistema de monitoramento sobre as métricas que são levadas em consideração pelos eventos e pela ativação. Se o Coordinator Service verificar que o evento deve ser disparado, ele realiza chamadas para os componentes Agent da Área de Sensor Corporal, para executar as ações do evento identificado.

O Forecast Service é o componente responsável para fornecer a possibilidade de estimar o valor de um conjunto de métricas em um quadro de observação futuro com base nos dados de monitoramento. Para verificar as previsões com base nas métricas monitoradas, o Coordinator Service recupera continuamente as definições das métricas preditivas e o quadro a ser previsto, bem como os dados de monitoramento dessas métricas, e as usa como entrada para o Forecast Service. Este retorna o valor da predição de cada métrica desejada. Usando esses dados, o Coordinator Service decide se a execução de qualquer ação no ambiente é necessária. 


\section{Resultados preliminares}

O quadro proposto neste artigo foi aplicado a um contexto real. A ideia consiste em uma adaptação do modelo arquitetônico no jogo wearable descrito em [Rocha et al. 2015], para testar a eficiência de nossa proposta. Também foi feita uma simulação usando um banco de dados. Nosso objetivo é verificar se o aspecto preditivo da ASAPe pode ser usado em um contexto real e se pode fornecer um serviço eficiente, eficaz e em tempo real de processamento de dados. Também queremos verificar a taxa de sucesso dos algoritmos utilizados em nossa arquitetura.

\subsection{Estudo de caso: Heart Wars}

O jogo "Heart Wars"permite unir a realização de uma atividade física com o lúdico, envolvendo áreas como saúde, fitness e jogos. A história do jogo acontece em um cenário fictício, onde um exército de corações é guiado por um personagem chamado Heart, o bárbaro. Os batimentos medidos devem permanecer na área verde, como na Figura 2. Se o jogador está registrando uma frequência cardíaca muito alta, a faixa entra na área vermelha e a pontuação diminui. O mesmo ocorre se o batimento cardíaco for reduzido e a faixa entrar na área azul. Neste contexto, ASAPe poderia ajudar com a verificação do batimento cardíaco antes de obter demasiado acelerar ou não avaliar o mínimo, para manter a faixa na área verde.

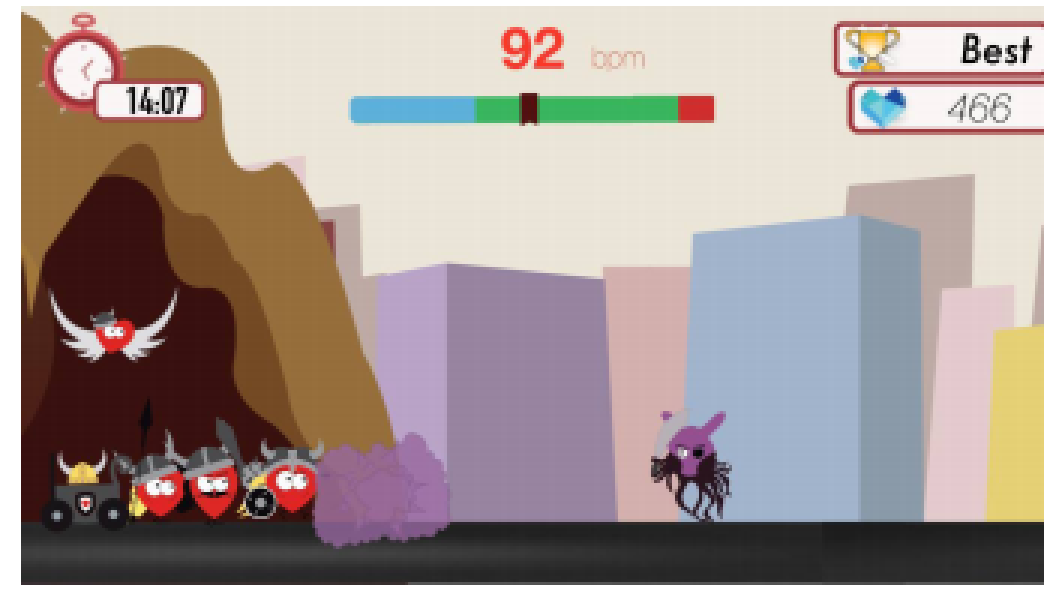

Figura 2. Imagem de jogo Heart Wars

[Rocha et al. 2015, p. 4]

Nestas experiências, fizemos medições reais. A métrica avaliada foi a acurária, pois é o fator que indica a exatidão dos resultados. Nossos parâmetros de sistema incluem um smartwatch $L G G$ Watch $R$, com um sensor de frequência cardíaca embutido. Os parâmetros de workload das medidas reais incluem séries de um exercício chamado burpee por 2 minutos, medindo o batimento cardíaco a cada 10 segundos, totalizando 14 medidas. Foi avaliada a previsão para verificar a exatidão dos valores obtidos com a ASAPe e, em seguida, utilizamos duas métricas para avaliar a precisão: Root-meansquare error (RMSE) e Mean absolute percentage error (MAPE).

Considere um conjunto de 14 amostras, para ambos os algoritmos, escolhemos um conjunto de treinamento composto por sete medidas e depois, classificamos os demais. Ambas as métricas mostraram que os valores preditos eram semelhantes aos dados 
verdadeiros. Os resultados do ARIMA foram mais precisos do que os do SVR. O número obtido com RMSE de ARIMA foi 4.3077 e 4.3846 para SVR. Ambos os algoritmos tiveram bons resultados, com uma baixa taxa de erro, mas ARIMA teve o menor número. $\mathrm{O}$ resultado obtido com a equação de MAPE foi 0,0152 para ARIMA e 0,0180 para SVR. Novamente a taxa de erro foi pequena, mas ARIMA obteve melhores resultados do SVR.

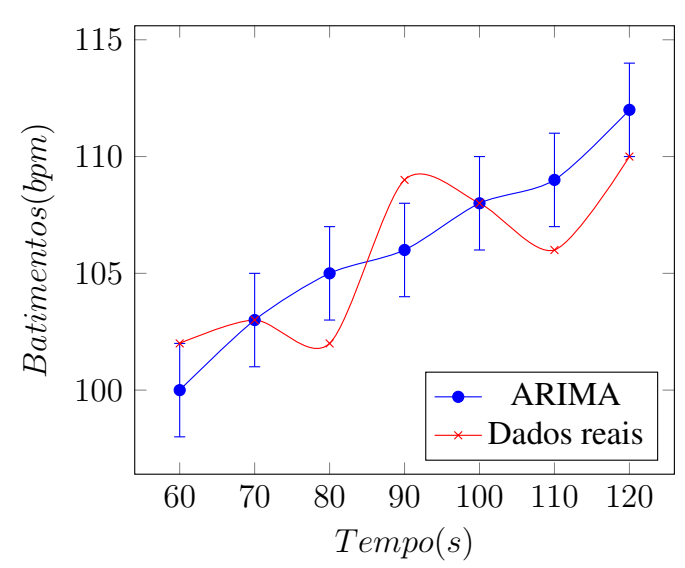

(a) Resultados do ARIMA

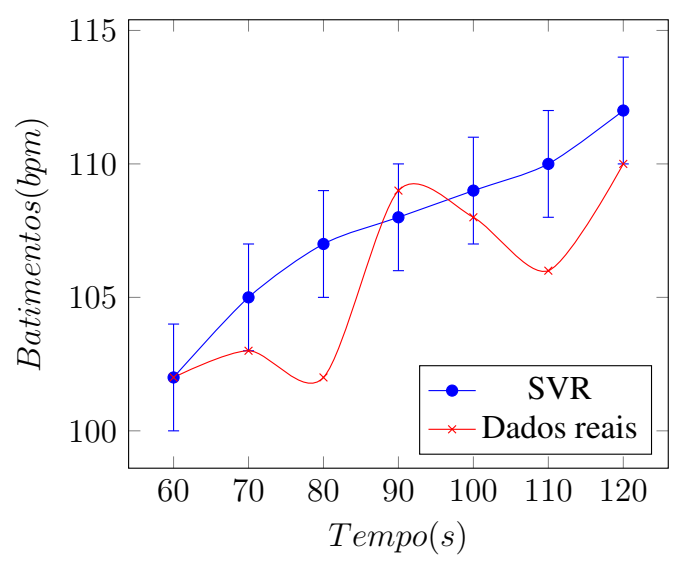

(b) Resultados do SVR

Figura 3. Resultados da avaliação do caso de estudo

Como discutido anteriormente, o Forecast Service decide o método a ser usado para fazer a predição. Nesse caso, ARIMA foi escolhido para prever o intervalo de tempo, pois como pode ser observado na Figura 3, foi o que obteve melhores resultados em termos de acurácia.

\subsubsection{Simulação}

Para essa avaliação, usamos o banco de dados MIT-BIH dataset [Glass et al. 2012], um conjunto de medidas de frequência cardíaca, composto por quatro séries temporais de 1800 dados cada um. As medidas, em unidades de batimentos por minuto (bpm), foram retiradas de dois indivíduos praticando atividades físicas durante 15 minutos, medida em cada 0,5 segundo.

Considere dois conjuntos de 1800 amostras cada um. Foram usados 33\% (594 amostras) para testes e 66\% (1188 amostras) para treinamento e validação para SVR e ARIMA. Utilizamos os dados de validação (compostos por $30 \%$ dos dados de treinamento) para sintonizar todos os hiper parâmetros para SVR e para o modelo ARIMA, usamos o procedimento Hyndman e Khandakar [Hyndman et al. 2007], obtendo os resultados vistos nas Figuras 4 e 5.

Conforme observado nas Figuras 4 e 5, tanto SVR como ARIMA apresentaram valores semelhantes aos dados verdadeiros. Nas simulações, o número obtido com RMSE do primeiro conjunto de dados (Figura 4) de ARIMA foi de 56,44 e 54,90 para SVR. Ambos tiveram bons resultados, mas o SVR apresentou o menor número de erros. No segundo (Figura 5), ARIMA obteve 21.13 e SVR, 43.27. O resultado obtido com a equação de MAPE no primeiro conjunto de dados foi de 6,71 para ARIMA e 6,55 para SVR. No segundo, o resultado foi de 3.77 para ARIMA e 5.22 para SVR. 


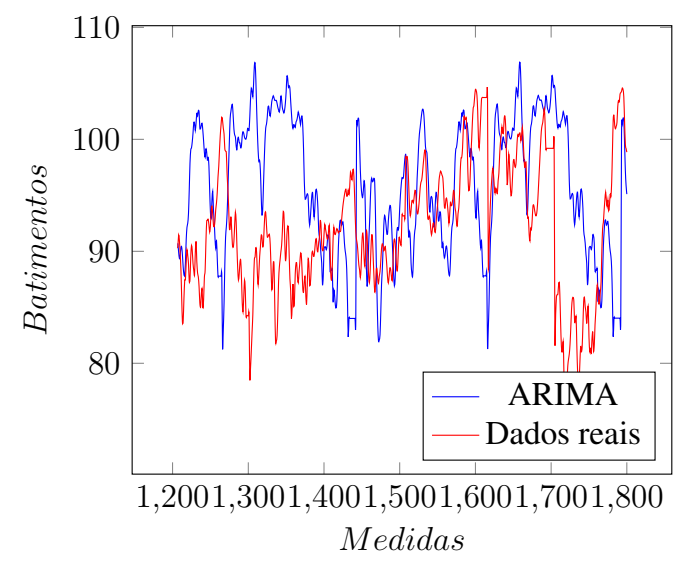

(a) Resultados do ARIMA

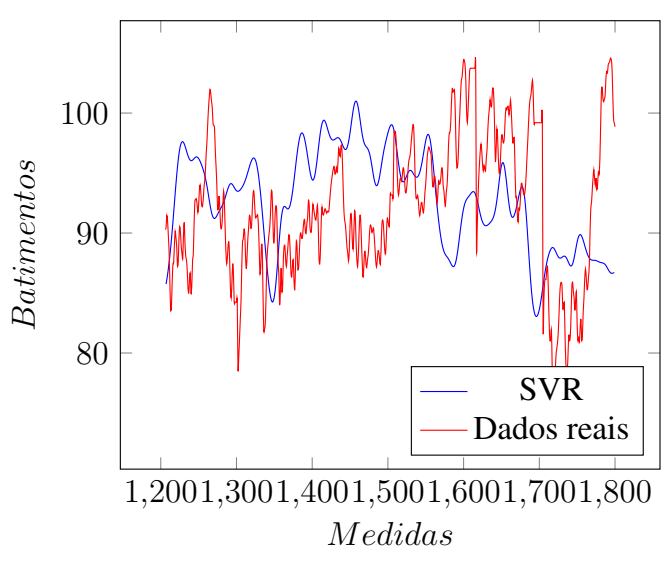

(b) Resultados do SVR

Figura 4. Resultados da ASAPe para a série 1.

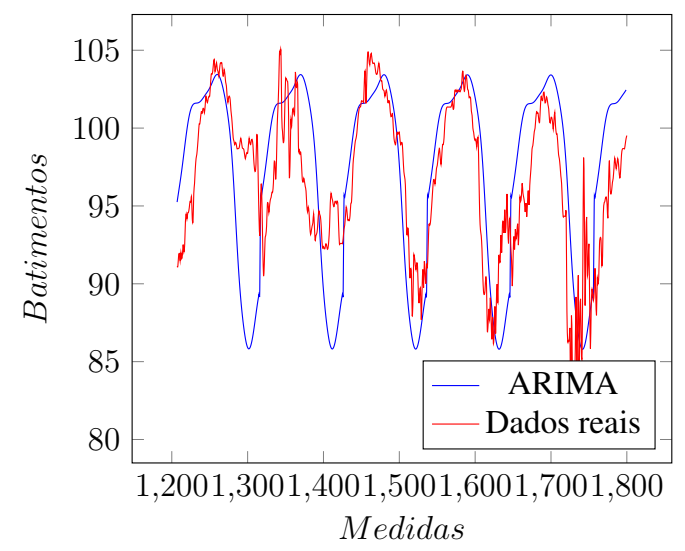

(a) Resultados do ARIMA

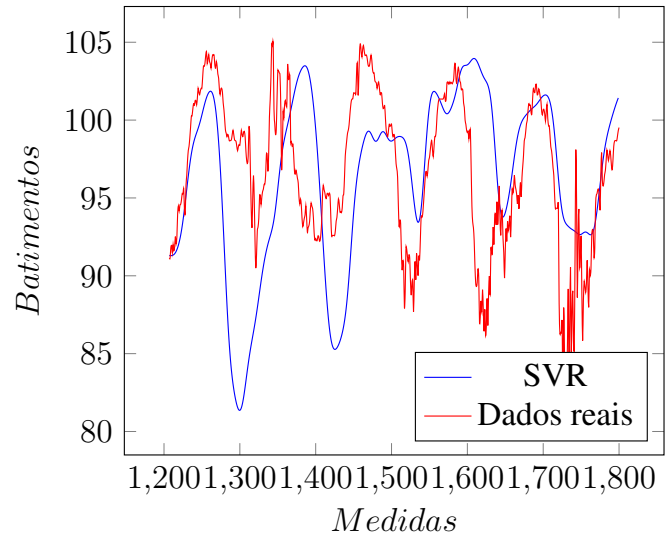

(b) Resultados do SVR

Figura 5. Resultados da ASAPe para a série 2.

\section{Conclusão}

Nossa proposta consiste em uma arquitetura para predição em infraestruturas wearable. Este tipo de aplicação pode ajudar no desenvolvimento de muitos sistemas vestíveis que necessitam da característica preditiva. Poderia também ser adaptado aos sistemas existentes, para melhorar a previsão destes ou, quando não tem uma camada de predição, obter esta característica. A avaliação mostrou que a ASAPe é útil em contextos de recolha de dados em tempo real.

Como trabalhos futuros, vamos incluir a análise de mais dados em diferentes ambientes para obter dados confiáveis sobre precisão. Além disso, planejamos testar diferentes sensores e dispositivos para verificar a forma como a ASAPe funciona com uma variedade de dados. Pretendemos incorporar na ASAPe a outras aplicações para testar sua portabilidade e a precisão em outros contextos. Finalmente, outros recursos podem ser adicionados à ASAPe, como eficiência energética e segurança. 


\section{Agradecimentos}

Michelle Cacais agradece a CAPES pela bolsa de mestrado (processo \#1589313). Danielo G. Gomes agradece ao CNPq pela bolsa de produtividade PQ \#311878/2016-4 e projeto Universal \#432585/2016-8.

\section{Referências}

Abdali-Mohammadi, F., Bajalan, V., and Fathi, A. (2015). Toward a fault tolerant architecture for vital medical-based wearable computing. Journal of medical systems, 39(12):149.

Chen, M., Zhang, Y., Li, Y., Hassan, M. M., and Alamri, A. (2015). Aiwac: Affective interaction through wearable computing and cloud technology. IEEE Wireless Communications, 22(1):20-27.

Conjeti, S., Singh, R. R., and Banerjee, R. (2012). Bio-inspired wearable computing architecture and physiological signal processing for on-road stress monitoring. In Proceedings of 2012 IEEE-EMBS International Conference on Biomedical and Health Informatics, pages 479-482. IEEE.

Forkan, A. R. M. and Khalil, I. (2016). A probabilistic model for early prediction of abnormal clinical events using vital sign correlations in home-based monitoring. In Pervasive Computing and Communications (PerCom), 2016 IEEE International Conference on, pages 1-9. IEEE.

Galzarano, S., Giannantonio, R., Liotta, A., and Fortino, G. (2016). A task-oriented framework for networked wearable computing. IEEE Transactions on Automation Science and Engineering, 13(2):621-638.

Gheith, A., Rajamony, R., Bohrer, P., Agarwal, K., Kistler, M., Eagle, B. W., Hambridge, C., Carter, J., and Kaplinger, T. (2016). Ibm bluemix mobile cloud services. IBM Journal of Research and Development, 60(2-3):7-1.

Glass, L., Hunter, P., and McCulloch, A. (2012). Theory of heart: biomechanics, biophysics, and nonlinear dynamics of cardiac function. Springer Science \& Business Media.

Gope, P. and Hwang, T. (2016). Bsn-care: A secure iot-based modern healthcare system using body sensor network. IEEE Sensors Journal, 16(5):1368-1376.

Hiremath, S., Yang, G., and Mankodiya, K. (2014). Wearable internet of things: Concept, architectural components and promises for person-centered healthcare. In Wireless Mobile Communication and Healthcare (Mobihealth), 2014 EAI 4th International Conference on, pages 304-307. IEEE.

Hyndman, R. J., Khandakar, Y., et al. (2007). Automatic time series for forecasting: the forecast package for $R$. Number 6/07. Monash University, Department of Econometrics and Business Statistics.

Jaimes, L. G., Gagneja, K., Akbaş, M. İ., and Vergara-Laurens, I. J. (2017). Future stress, forecasting physiological signals. In Computing and Communication Workshop and Conference (CCWC), 2017 IEEE 7th Annual, pages 1-5. IEEE. 
Kan, V., Fujii, K., Amores, J., Zhu Jin, C. L., Maes, P., and Ishii, H. (2015). Social textiles: Social affordances and icebreaking interactions through wearable social messaging. In Proceedings of the Ninth International Conference on Tangible, Embedded, and Embodied Interaction, pages 619-624. ACM.

Kang, J. J., Adibi, S., Larkin, H., and Luan, T. (2015). Predictive data mining for converged internet of things: A mobile health perspective. In Telecommunication Networks and Applications Conference (ITNAC), 2015 International, pages 5-10. IEEE.

Liu, Y. and Zhou, G. (2012). Key technologies and applications of internet of things. pages 197 - 200, Zhangjiajie, Hunan, China. IEEE.

Molina-Markham, A., Peterson, R., Skinner, J., Yun, T., Golla, B., Freeman, K., Peters, T., Sorber, J., Halter, R., and Kotz, D. (2014). Amulet: a secure architecture for mhealth applications for low-power wearable devices. In Proceedings of the 1st Workshop on Mobile Medical Applications, pages 16-21. ACM.

Nguyen, L. N. N., Rodríguez-Martín, D., Català, A., Pérez-López, C., Samà, A., and Cavallaro, A. (2015). Basketball activity recognition using wearable inertial measurement units. In Proceedings of the XVI International Conference on Human Computer Interaction, page 60. ACM.

Pels, T., Kao, C., and Goel, S. (2014). Fatbelt: motivating behavior change through isomorphic feedback. In Proceedings of the adjunct publication of the 27th annual ACM symposium on User interface software and technology, pages 123-124. ACM.

Rocha, U., Oliveira, D., Carvalho, L., Moreira, L., and Viana, W. (2015). Heart wars: um exergame de batalha controlado por dispositivos vestiveis. In Anais do XV Workshop de Informática Médica (WIM), pages 1-10, Porto Alegre, RS, Brasil. SBC.

Salehizadeh, S., Noh, Y., and Chon, K. (2016). Heart rate monitoring during intense physical activities using a motion artifact corrupted signal reconstruction algorithm in wearable electrocardiogram sensor. In Connected Health: Applications, Systems and Engineering Technologies (CHASE), 2016 IEEE First International Conference on, pages 157-162. IEEE.

Spelmezan, D. (2012). An investigation into the use of tactile instructions in snowboarding. In Proceedings of the 14th international conference on Human-computer interaction with mobile devices and services, pages 417-426. ACM.

Uddin, M., Salem, A., Nam, I., and Nadeem, T. (2015). Wearable sensing framework for human activity monitoring. In Proceedings of the 2015 workshop on Wearable Systems and Applications, pages 21-26. ACM.

Wang, K., Wang, Y., Sun, Y., Guo, S., and Wu, J. (2016). Green industrial internet of things architecture: an energy-efficient perspective. IEEE Communications Magazine, 49. 